\title{
The Sustainability of Knowledge-Sharing Behavior Based on the Theory of Planned Behavior in Q\&A Social Network Community
}

\author{
Xin Feng $\mathbb{D}^{1},{ }^{1}$ Lijie Wang $\left(\mathbb{D},{ }^{2}\right.$ Yue Yan $\mathbb{D},,^{3}$ Qi Zhang $\mathbb{D},,^{2}$ Liming Sun $\mathbb{D}^{4},{ }^{4}$ Jiangfei Chen $\mathbb{D}^{5},{ }^{5}$ \\ and $Y e \mathrm{Wu}^{6}{ }^{6}$ \\ ${ }^{1}$ School of Economics and Management, Yanshan University, Qinhuangdao 066004, China \\ ${ }^{2}$ Business School, University of International Business and Economics, Beijing 100029, China \\ ${ }^{3}$ Huaxin College, Hebei GEO University, Shijiazhuang 050031, China \\ ${ }^{4}$ School of Language and Culture, Hebei GEO University, Shijiazhuang 050031, China \\ ${ }^{5}$ Miami University, Middletown 45042, OH, USA \\ ${ }^{6}$ School of Journalism and Communication, Beijing Normal University, Beijing 100875, China
}

Correspondence should be addressed to Lijie Wang; wanglijie@uibe.edu.cn

Received 13 May 2021; Accepted 10 June 2021; Published 19 June 2021

Academic Editor: Xuzhen Zhu

Copyright (c) 2021 Xin Feng et al. This is an open access article distributed under the Creative Commons Attribution License, which permits unrestricted use, distribution, and reproduction in any medium, provided the original work is properly cited.

With the update and iteration of Internet technology, the socialized Q\&A (question-and-answer) platform realizes the crossborder dissemination of knowledge with the main purpose of disseminating and sharing knowledge. Zhihu, as a knowledgesharing platform that relies on the user-generated content model to maintain operation and the strong willingness of users to share knowledge, plays a key role in the development of the community. Currently, social Q\&A platforms are facing problems such as low user participation rate and gradual decrease in the number of active users. It is very important and urgent to explore the factors that affect users' willingness to share knowledge. In response to this problem, this paper builds a theoretical model of the factors that are affecting users' willingness to share knowledge and uses questionnaire research methods to conduct research design and collect sample data and uses methods such as correlation analysis and structural equation modeling to verify the model and hypothesis. The research results show that the theoretical model of planned behavior has strong explanatory power and selfefficacy and material rewards have a positive effect on knowledge-sharing attitudes. Finally, according to some research results, this paper shows that, with the change of time, young people have different needs for knowledge sharing than before, while realizing self-worth through sharing experience, and we also hope to protect own interests and hope that there are more factors to encourage more users to share their knowledge and experience. Therefore, we propose that the platform can use incentive mechanisms to promote knowledge sharing while helping sharers realize their self-worth, improve the existing functions of the platform, or carry out activities to encourage users to participate, so as to achieve the purpose of knowledge sharing and maintain the operation of the Zhihu platform.

\section{Introduction}

1.1. Research Significance. More and more domestic and foreign researchers have begun to focus on the study of knowledge sharing; in whether traditional community or virtual community, information flow and knowledge sharing play an important role in the development of communities.
Through the analysis of Zhihu users' attitudes, motivations, and other factors affecting knowledge sharing, a knowledge community model that provides the sustainability of knowledge-sharing behaviors is constructed. Researching and summarizing the influencing factors of the willingness of community users to share knowledge can guide people to continue knowledge sharing and information exchange in 
the Zhihu community and propose suggestions to promote community knowledge sharing to the socialized questionand-answer community represented by Zhihu.

1.2. Research Purpose. Whether in traditional communities or virtual communities, the flow of information and the development of knowledge communities are crucial. At present, Zhihu has become a typical representative of the knowledge community. This article uses Zhihu users as an example to study and analyze the factors that affect the knowledge-sharing willingness of users in the knowledge community. Based on the summary of domestic and foreign research, this article combines the theory of planned behavior (TPB) with Zhihu community users as the research object and discusses the influencing factors of Zhihu users' willingness to share. This research constructs a theoretical research model, defines research variables and proposes hypotheses, designs and develops measurement tools and questionnaires, conducts data analysis and verification of research hypotheses on the questionnaire survey data, and then discusses and researches the results.

\subsection{Status of Domestic and Foreign Research}

1.3.1. Foreign Research Trends. Research on knowledgesharing platforms by foreign scholars started early. Harper et al. [1] believed that the knowledge-sharing platform does not limit the types of questions and the domains they belong to and allows users to ask and answer a wide range of questions without violating the law. This widens the scope of users' discussions, and the platform can contact more users with various types of questions. The platform becomes more flexible and active, and users have the possibility of reaching more knowledge. Driven by knowledge and curiosity, users will continue to be active. Choi and $\mathrm{Yi}$ [2] took the respondents of the Yahoo community as the research object and studied the factors that affect their knowledge-sharing behavior. It found that altruism, ideology, self-efficacy, and pleasure significantly positively affect the willingness to share knowledge. These factors belong to the intrinsic motivation of behavior, altruist for personality beliefs, and likes to help others. In terms of ideology, when people's opinions, values, etc., collide with other opinions, whether it is a good collision or a vicious collision, it is more likely to lead to the generation of knowledge-sharing behavior. Knowledge-sharing behavior is also related to the user's history of success or failure. The more success stories a person has, the higher the sense of accomplishment, the higher the degree of trust in knowledge, and the higher the probability of knowledge-sharing behavior, which is the positive impact of self-efficacy. Emotionally, a happy mood is more likely to cause people to share behaviors, not just knowledge-sharing behaviors, and a cold mood is not easy to produce knowledge-sharing behaviors and is more likely to produce talk and silent behaviors. Cronk [3] combines social capital theory with a positive emotional tone to form a new comprehensive model. The experimental results show that positive emotional tone, trust, and common vision will have a positive impact on knowledge sharing. At the same time, the role of trust and social interaction on knowledge sharing is regulated by the positive emotional tone. The idea of trust and interaction comes from emotional judgments, and positive emotions promote the generation of trust and interactive ideas. In the theory of social capital, the one with the other in a friendly and noncompetitive cooperative relations will be more prone to knowledge sharing.

As the influence of socialized knowledge sharing gradually grows, researchers have made relevant research on the influence of knowledge-sharing behavior. Alexander and Nick [4] believed that self-interest, community culture, and trust among members are positively related to the willingness to share knowledge. When there are sufficient selfinterest conditions, such as answering questions to earn reward points or accumulate personal fame, such conditions will lead some users to actively answer questions; when answering questions becomes a very common phenomenon in community platforms, users are accustomed to answering questions to each other and even indulging in the joy of answering questions, which can also be called community culture. This is undoubtedly the cultural environment conducive to the emergence of knowledge-sharing behavior. Meng et al. [5] found that self-efficacy, trust, and result expectations are the main factors affecting knowledge sharing in virtual communities; on the virtual social platform, people do not know each other. While seeking knowledge to get a certain answer, another question will follow, which is the correctness of the answer. This scenario is very common in virtual communities, and sometimes wrong answers will occur. Very bad influences, such as asking about the database addition operation, when someone inadvertently answered the database deletion operation in a joke, will cause very serious consequences and make the trust between people in the virtual community disappear. When there are certain official certifications or correct certification marks, knowing the expected results will make it easier for people to accept and leave a trust mark in the virtual community, which is conducive to long-term active knowledge-sharing behavior. According to the TPB, Hassandoust et al. research [6] on members' knowledgesharing intention found that attitude and perceived behavior control have a significant impact on knowledge-sharing intention, while subjective norms have no significant impact on knowledge-sharing intention. Attitude drives behavior, and knowledge-sharing behavior actually occurs when members feel the need to share knowledge. When members are subjectively required to share knowledge, it is more likely to become an imposed burden or task, and only a small probability of good knowledge-sharing behavior will occur. According to the research on the influence of social network relationship and TPB on knowledge-sharing intention and behavior, Chen et al. [7] showed that subjective norms, students' attitude, self-efficacy, and social relations have a significant positive correlation with knowledge-sharing intention and indirectly influence knowledge-sharing behavior through knowledge-sharing intention. Tao [8] constructed a virtual community knowledge-sharing influencing factor model based on social cognition theory and further explored 
self-efficacy, trust, perceived compatibility, and perceived relative advantages. The influence mechanism of these four factors on knowledge-sharing behavior has been found to have a significant impact on knowledge-sharing behavior. Trust positively affects self-efficacy, and there is a significant relationship between perceived relative advantage and perceived compatibility.

1.3.2. Domestic Research Trends. Chen Juan, based on the theory of self-determination, used regression analysis to study the influencing factors of the user experience. The results show that the improvement of visual attractiveness and demand satisfaction will enhance the user experience, and emotions play a moderating role in it. Vision brings the user an intuitive feeling, which is the first impression during the experience, and the comfortable visual effect improves the user experience. Inspired by the six-degree segmentation theory, Liu and Jia proposed a knowledge contribution behavior research model suitable for Zhihu users on the basis of the knowledge-sharing cross graph [9]. She believes that trust, incentive system, community culture, and results are expected to positively affect the knowledge-sharing behavior of Zhihu users. The trust is high; with the question of answering rewards, the official certification mark, and celebrities answering, these will be more popular in the community.

According to the TPB, Li Zhihong introduced social cognition theory and social exchange theory and constructed theoretical models to obtain trust, result expectation, self-efficacy, and altruism, which were significantly related to the willingness of members to share knowledge. Based on the theory of social capital, Zhang Nai and Zhou Nianxi found that social interaction in virtual communities can have a positive impact on knowledge-sharing behavior. Based on action control theory and TPB, Jiang Peizhen found that subjective norms, self-efficacy, and users' attitudes towards knowledge sharing are significantly positively correlated, and knowledgesharing attitudes have a direct positive impact on knowledgesharing behavior. Liu et al. [10] proceeded from the social and cultural dimensions and individual psychological dimensions and proposed the influence of personal outcome expectations, self-efficacy, relationships, and other influencing factors on the willingness to share knowledge in virtual communities. According to research, personal outcome expectations, relationships, etc. have a significant impact on the willingness to share knowledge in virtual communities, while self-efficacy has no significant impact on personal outcome expectations. Zhao Yuxiang integrated social exchange theory, technology acceptance model theory, social capital theory, etc., constructed an integrated theoretical model, and proposed three factors that affect users' use of blogs: perceived usefulness, perceived ease of use, and exchange costs.

\section{The Definition of Theoretical Concepts}

\subsection{Theory of Planned Behavior}

2.1.1. Theory of Planned Behavior's Concept. The theory of planned behavior (TPB) is derived from the Theory of Reasoned Action (TRA), which emphasizes that human behavior is based on rational volitional control and mainly determined by attitudes and subjective norms. The TPB was put forward by Icek Ajzen. Ajzen believed that all factors that may affect behavior indirectly influence the performance of behavior through behavioral intention. Fu [11] conducted an empirical study on the influencing factors of individuals' and organizations' willingness to explore knowledge sharing and found that knowledge sharing is significantly influenced by organizational image and altruism to a large extent. In conclusion, a large number of studies have proved that the TPB is applicable to the study of knowledge sharing.

2.1.2. TPB's Research Status. Based on TPB, Ajzen [12] put forward the theory of decomposing planned behavior. They believe that there are multiple concepts in attitude, subjective norms, and perceptual behavior control, decomposing attitudes into emotional and instrumental, and dividing subjective norms into command elements and descriptive elements; perceptual behavior control includes two aspects of self-efficacy and controllability. According to the research on the TPB, Keats et al. [13] pointed out that, when predicting behavior and intention, the occurrence of individual behavior is affected by the expectation of others. Zahra and Mohammad and Chen [14, 15] studied knowledge-sharing behavior using TRA or TPB theoretical model, and the research showed that knowledge-sharing intention has a direct influence on knowledge-sharing behavior. Bello and Oyekunle [16] studied that knowledgesharing behavior is influenced by attitude, intention, and motivation by using the TPB. Knowledge-sharing attitude is significantly related to intention, and the knowledgesharing intention is also significantly related to behavior. Jin et al. [17] used TRA to study the process of knowledge sharing in the communication industry and found that perceived knowledge ownership and material incentives have a positive impact on individuals' willingness and behavior of knowledge sharing. Based on the TPB, Zhao [18] integrated the key variables of expectation confirmation theory, social cognition theory, and social capital theory, extended the original variables of planned behavior theory, and constructed a new theoretical model of planned behavior. Based on this theoretical model, this study will build a new theoretical model to study the sustainability of community users' knowledge sharing.

\subsection{Knowledge Sharing}

2.2.1. The Concept of Knowledge Sharing. Knowledge sharing is the most complicated link in all aspects of knowledge management, and it is also the key step for successful knowledge management. Knowledge sharing is also a kind of dissemination behavior; from the contribution and flow of knowledge to the adoption, digestion, and absorption of knowledge, people can acquire knowledge from others through this behavior. The concept of knowledge sharing is not entirely uniform. Pilerot [19] put forward that knowledge sharing can be explained and understood by words such as transfer and giving. Tong et al. [20] defines 
knowledge sharing as the process of exchanging tacit knowledge and creating new knowledge among friends, families, organizations, or communities. Hao et al. [21], a domestic scholar, pointed out that easiness is the basis of knowledge-sharing behavior, and it is the marginal increasing utility of knowledge that promotes the emergence of the knowledge-sharing phenomenon.

2.2.2. Influencing Factors of Knowledge Sharing. The research results of Chan et al. [22] found that, in virtual communities, users' sense of community, helpfulness, and image finally promoted members' knowledge sharing. In the degree of knowledge sharing, time and interest in discussion topics played a moderating role. Koh and Kim [23] found that, by encouraging knowledge sharing among virtual community users, virtual community providers could enhance community activity and participation, enhance the business value of the community, and finally establish the loyalty of virtual community members to virtual community providers. Based on social exchange theory, Yang [24] verified that reciprocity and trust had positive effects on knowledge-sharing behavior by using regression analysis method. Based on rational behavior theory, Yang [25], combined with incentive theory and social exchange theory as a supplement, studied the mechanism of knowledgesharing willingness of users who have used social Q\&A websites and found that altruism and reciprocity have a positive effect on knowledge-sharing attitude; sharing attitude and self-efficacy have a significant effect on knowledgesharing willingness, while subjective norms have not shown a significant effect on willingness in this research. Trust also has a significant positive effect on sharing attitude and willingness.

\section{Construction of Theoretical Model and Research Hypothesis}

3.1. Construction of Theoretical Model. In the research of behavior prediction, many scholars and experts believe that behavioral intention is closer to behavior than attitude, belief, and feeling. Therefore, understanding an individual's intention for a specific behavior is an important prerequisite for predicting whether an individual will perform the behavior. Fishbein and Ajzen [26] believe that attitude, subjective norms, and cognition of behavior control are important psychological factors that directly affect behavioral intention. On this basis, Zhang [27] put forward four influencing factors of knowledge sharing among individuals in mobile Internet, which are expected organizational reward, reciprocal benefit, knowledge self-efficacy, and altruistic beliefs, and established the following model (Figure 1).

Based on Figure 1, influencing factors of group recognition and material reward are added which are shown in Figure 2. Wang et al. [28] believe that group recognition is an important factor for users to build trust and increase influence in social networks. According to the social exchange theory of Blau and Li [29], all human behaviors are dominated by a certain exchange activity that can bring rewards and remunerations; therefore, all social activities of human beings can be summed up as an exchange. At present, China's knowledge-based communities often use material rewards to control the behavior of this kind of spontaneous knowledge sharing.

This research is guided by the TPB, constructs a theoretical model of Zhihu users' sharing willingness, studies the influence of attitude and perceived behavior control variables on behavior willingness, and studies the characteristics of Zhihu community users. Introduce reciprocity, group recognition, self-efficacy, altruistic beliefs, and material rewards, and study the influence of these variables on the knowledge-sharing willingness.

\subsection{Research Hypothesis Establishment.}

(1) The influence of reciprocity on knowledge-sharing attitude. Reciprocity as a benefit also inspires responsibility and trust among individuals. Studies as early as the 1990s have found that reciprocity is a significant motivator for knowledge sharing [30]. A large number of subsequent studies have also demonstrated the impact of reciprocal benefits on knowledge sharing. For example, Bock and Kim [31] elaborated the impact of expected reciprocal relationship on knowledge-sharing attitude. Through empirical research, Lin [32] found that reciprocal benefits significantly affected employees' attitude and intention of knowledge-sharing behavior. Therefore, this paper proposes the following hypotheses:

\section{H1: reciprocity positively influences knowledge-} sharing attitude.

(2) The influence of group recognition on knowledgesharing attitude. Group recognition is an important factor that affects trust and social influence in social networks. Group recognition is a measure of a person's degree of social recognition. When group recognition is low, it means that the person's social credibility is low. When group recognition is high, it means that this person is recognized by the majority of people in society and also recognizes the knowledge shared by him.

\section{H2: group recognition has a positive effect on} knowledge-sharing willingness.

(3) The influence of self-efficacy on willingness to share knowledge. Self-efficacy will affect individuals' intention and cognitive ability to do something. Users' subjective judgment formed after knowledge sharing is their self-assessment of their ability to provide valuable knowledge to other users. The perception of self-efficacy plays an important role in influencing people's motivation and behavior. Runhaar and Sanders [33] showed that self-efficacy had a significant predictive effect on knowledge-sharing behavior, and people with high self-efficacy had higher 
willingness and behavior for knowledge sharing. The research of Hsu et al. [34] found that self-efficacy, as intrinsic motivation, can directly or indirectly affect the willingness to share knowledge in virtual communities. Individuals with high self-efficacy have stronger willingness to share knowledge. Therefore, the following hypothesis is proposed:

H3: self-efficacy has a positive influence on willingness to share knowledge.

(4) The influence of altruistic beliefs on knowledgesharing willingness. In social question-and-answer communities, a large number of people answer questions for other users as contributors, choosing to ask questions and then expounding their own views. Gan et al. [35] found that altruistic beliefs can promote users' knowledge-sharing behavior in the question-and-answer community. The typical network altruistic behavior is that the respondent, as the contributor, answers the questions of others, which needs to meet the needs of the recipient when the specific value is met. Therefore, this paper proposes the following hypotheses:

H4: altruistic beliefs have a positive effect on knowledge-sharing willingness.

(5) The influence of material rewards on knowledgesharing willingness. To complete a high-quality article, users need to spend a lot of time editing text or images or even models. Knowledge sharing requires efforts, and if efforts are proportional to returns, users' willingness to share knowledge can be promoted. High-quality knowledge sharing requires early accumulation and investment of a lot of time, energy, and money. Therefore, the following assumptions are listed:

H5: material rewards have a positive effect on knowledge-sharing willingness.

\subsection{Questionnaire Design}

3.3.1. Questionnaire Design Instructions. In this research, Likert five-level scale was used to measure variables. The questionnaire was composed of three parts:

The first part is the introduction, which clarifies the purpose of this research.

The second part is the basic personal information, which mainly includes the time, age, gender, and education level of the respondents on Zhihu.

The third part is the measurement question of the variables in this research. The respondents are required to give a grade of 1-5 based on their personal experience, and the corresponding levels are very disapproving, disapproving, indifferent, approving, and very approving. Table 1 shows the questionnaire variable design, and the questionnaire item design is shown in Table 2.
3.3.2. Questionnaire Item Design. Questionnaire item design is provided in Table 2 .

\section{Data Collection and Analysis}

4.1. Sample Profile and Descriptive Analysis. In this research, the data were collected by questionnaire, and the survey methods used in this questionnaire are all distributed in the form of network questionnaire. It is mainly distributed to the respondents in the form of links and two-dimensional codes. The regulations for the respondents are user of Zhihu. Specifically, since we defined the specific users of the questionnaire survey as college students, the age of the users can be confirmed as the young user group. At the same time, in order to ensure the randomness of the questionnaire, we randomly distributed questionnaires on major social platforms to call on more young user groups to participate in the survey. Among them, we linked the questionnaire to some Zhihu users in Zhihu community randomly and randomly selected and distributed questionnaires in multiple groups on Sina Weibo, QQ, and WeChat. A total of 280 questionnaires were distributed, and 271 valid questionnaires were collected. The specific questionnaire survey results are shown in Table 3.

Based on the descriptive statistics of the above survey results, it can be concluded that the survey objects have the following characteristics:

(1) Service life of Zhihu: $18.5 \%$ of the respondents come to Zhihu for 1-6 months, $37.6 \%$ for $1-2$ years, $26.2 \%$ for 3-4 years, and $17.7 \%$ for more than 5 years, which ensures that the respondents are all users who have used Zhihu.

(2) Gender characteristics: the sample size of boys is 106 , accounting for $39.1 \%$ of the total sample. The sample size of female students is 165 , accounting for $60.9 \%$ of the total number of samples. Relatively speaking, females account for a large proportion.

(3) Age characteristics: most of the respondents are 21-30 years old, accounting for $74.9 \%$, mainly because most of the respondents are school students, so the age structure is more consistent.

(4) Education level: the target of the questionnaire is mainly college students, so the sample size of undergraduates is 187 , accounting for $69.0 \%$, those with junior college or below is $11.1 \%$, those with master's degree account for $12.9 \%$, and those with doctor's degree account for $7.0 \%$ of the total sample size.

4.2. Reliability and Validity Analysis of the Questionnaire. Firstly, this paper uses SPSS 25.0 to analyze the reliability of the survey data. Secondly, Excel, SPSS, and AMOS software were used to conduct validity analysis of the questionnaire and scale to verify the validity of the scale contents. Finally, the goodness of fit of the model was verified by confirmatory factor analysis. 


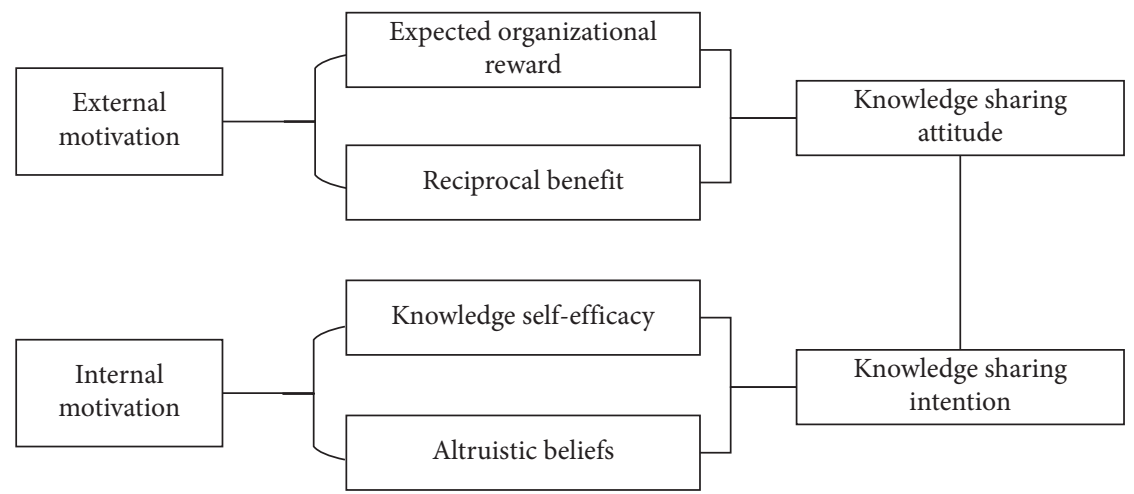

Figure 1: A theoretical model of influencing factors of individual knowledge sharing in the mobile Internet environment.

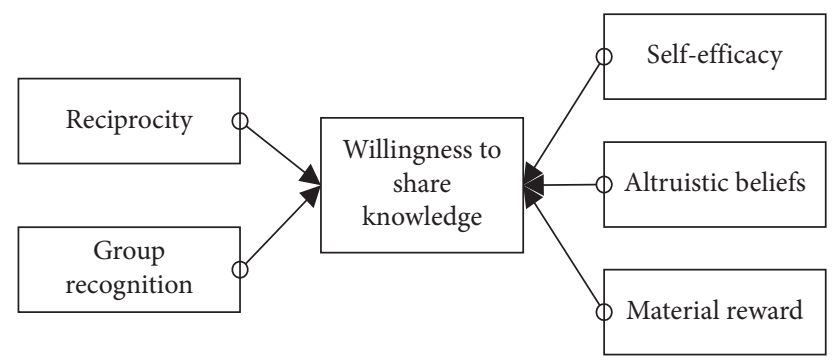

FIgURE 2: Theoretical model of Zhihu users' sharing willingness.

TABle 1: Questionnaire variable design.

\begin{tabular}{|c|c|c|}
\hline Variable & Variable definition & Reference \\
\hline Reciprocity & Respondents exchange knowledge contribution behaviors in the community & Shen et al. [36] \\
\hline Group recognition & $\begin{array}{c}\text { The trust established by users in the social network and the satisfaction after sharing } \\
\text { knowledge promote sharing behavior }\end{array}$ & Wang et al. [28] \\
\hline Self-efficacy & Personal confidence in their ability to provide knowledge & Hao et al. [21] \\
\hline Altruistic beliefs & Contributors choose to answer the questioner's questions & Gan et al. [35] \\
\hline Material rewards & An incentive to influential users for their efforts & $\begin{array}{l}\text { Atreyi Kankanhalli } \\
\text { et al. }\end{array}$ \\
\hline $\begin{array}{l}\text { Knowledge-sharing } \\
\text { willingness }\end{array}$ & $\begin{array}{l}\text { An individual's judgment of the subjective probability of taking knowledge-sharing } \\
\text { behavior, which reflects the individual's willingness for a specific behavior }\end{array}$ & Bock and Kim [31] \\
\hline
\end{tabular}

4.2.1. Reliability Analysis. Reliability analysis is mainly used to test the stability or consistency of the analysis results, that is, through multiple analysis of the questionnaire data, to observe whether the analysis results are consistent, in order to determine the authenticity or reliability of the empirical research results. Generally speaking, reliability tests are conducted in different time periods, among different respondents and raters. The higher the consistency of the final results is, the higher the reliability of the questionnaire is. Reliability can be divided into retest reliability, duplicate reliability, internal consistency reliability, and rater reliability. In this study, Cronbach's alpha reliability measurement method, which is widely used in previous studies and has a high degree of recognition, is adopted to conduct the reliability analysis of the questionnaire. Cronbach's alpha can fully reflect the implementation of the final scores of all grid question indicators in the scale. It can be seen from
Tables 4 and 5 that the overall reliability of these variables is 0.938 and the Cronbach- $\alpha$ coefficient of each variable is higher than the minimum standard 0.6, indicating that the scale used in this study has good reliability and internal consistency, and the design is reasonable.

4.2.2. Validity Test. Validity refers to the degree to which the measurement tool can accurately measure the required measurement characteristics, mainly including content validity and construction validity. Content validity means that the content of the scale is appropriate and representative, and the item distribution is reasonable, which can reflect the characteristics of the measurement indicators. For the content validity, this study mainly refers to the questionnaire items designed by some published articles to modify and invited teachers to evaluate the designed 
TABLE 2: Questionnaire item design.

\begin{tabular}{|c|c|c|}
\hline Variable & Item & Reference \\
\hline Reciprocity & $\begin{array}{l}\text { While answering other people's questions, I hope they can also answer my questions } \\
\text { When I share knowledge, I hope to get knowledge from the platform }\end{array}$ & Shen et al. [36] \\
\hline Group recognition & $\begin{array}{l}\text { I think sharing knowledge on the platform can be recognized by others } \\
\text { A user's trust in me is the recognition of me } \\
\text { Sharing useful knowledge will increase others' recognition of me }\end{array}$ & Wang et al. [28] \\
\hline Self-efficacy & $\begin{array}{l}\text { I think I have the ability to share knowledge in the Zhihu community } \\
\text { I think I have the ability to share knowledge in Zhihu community } \\
\text { I feel confident that I could provide something that the rest of the community found } \\
\text { valuable } \\
\text { The knowledge I share will be helpful to other users in the community }\end{array}$ & Hao et al. [21] \\
\hline Altruistic beliefs & $\begin{array}{c}\text { I want to share my knowledge, experience, insight or skills with others } \\
\text { I answer questions to help people in need }\end{array}$ & Gan et al. [35] \\
\hline Material rewards & $\begin{array}{c}\text { I hope to get substantial rewards after sharing knowledge } \\
\text { After sharing knowledge, I think it is very important to get the corresponding } \\
\text { commission } \\
\text { After sharing knowledge, I think the user's subscription is very important } \\
\text { I hope that sharing valuable knowledge can be rewarded } \\
\text { I hope that the shared knowledge will be rewarded when it is useful to others } \\
\text { A certain amount of pay and influence in the community can encourage me to share } \\
\text { knowledge }\end{array}$ & $\begin{array}{l}\text { Atreyi Kankanhalli } \\
\text { et al. }\end{array}$ \\
\hline $\begin{array}{l}\text { Knowledge-sharing } \\
\text { willingness }\end{array}$ & $\begin{array}{l}\text { I would like to share knowledge and information more frequently on the Zhihu } \\
\text { community } \\
\text { I am willing to often share my experience on the Zhihu community } \\
\text { I am willing to share my knowledge more frequently with other users } \\
\text { I am willing to comment and reply other users' answers } \\
\text { I would like to use the community's reply, comment and other features regularly }\end{array}$ & Bock and Kim [31] \\
\hline
\end{tabular}

TABLE 3: Descriptive statistics of knowledge-sharing willingness survey.

\begin{tabular}{|c|c|c|c|}
\hline Project & Category & Frequency & Percentage \\
\hline \multirow{4}{*}{ Time } & $1-6$ months & 50 & 18.5 \\
\hline & 1-2 years & 102 & 37.6 \\
\hline & 3-4 years & 71 & 26.2 \\
\hline & 5 years and above & 48 & 17.7 \\
\hline \multirow{2}{*}{ Gender } & Male & 106 & 39.1 \\
\hline & Female & 165 & 60.9 \\
\hline \multirow{4}{*}{ Age } & $0-20$ years old & 39 & 14.4 \\
\hline & 21-30 years old & 203 & 74.9 \\
\hline & $31-40$ years old & 25 & 9.2 \\
\hline & Over 40 years old & 4 & 1.5 \\
\hline \multirow{4}{*}{ Education level } & Junior college or below & 30 & 11.1 \\
\hline & Bachelor degree & 187 & 69.0 \\
\hline & Master's degree & 35 & 12.9 \\
\hline & Doctor's degree & 11 & 7.0 \\
\hline \multicolumn{2}{|c|}{ Total } & 271 & 100.0 \\
\hline
\end{tabular}

questionnaire and give relevant suggestions. The construction validity refers to the degree to which the actual test results explain the measurement index. As for the construction validity, this study will mainly test the construction validity of the questionnaire scale through factor analysis. Before factor analysis, KMO measure and Bartlett sphere test should be used to verify the validity of the questionnaire. Cerny and Kaiser [37] believed that studies showed that when KMO value was between 0.6 and 1, it was suitable for factor analysis, and when KMO value was below 0.6 , it was not suitable for factor analysis. In this paper, the factor analysis method was used to test the validity of the measurement model and scale and obtain the Bartlett sphere test and the KMO calculation result table. It can be seen from Table 6 that the significance level of the Chi-square value of Bartlett spherical test is 0.000 and $\mathrm{KMO}$ is 0.931 , indicating that the scale has good validity and is suitable for factor analysis.

As can be seen from Table 7, the factor loading is about 0.5 , indicating that the factor convergence effect is good. 
TABLE 4: Overall reliability analysis data.

\begin{tabular}{lcc}
\hline Cronbach's alpha variable & Cronbach's alpha based on standardized items & Number of items \\
\hline 0.938 & 0.939 & 20 \\
\hline
\end{tabular}

TABLE 5: Reliability analysis of each variable.

\begin{tabular}{lcc}
\hline Variable & Number of items & Cronbach $\alpha$ 's coefficient \\
\hline Material rewards & 6 & 0.895 \\
Knowledge-sharing willingness & 4 & 0.817 \\
Group recognition & 3 & 0.797 \\
Self-efficacy & 3 & 0.820 \\
Reciprocity & 2 & 0.786 \\
Altruistic beliefs & 2 & 0.690 \\
\hline
\end{tabular}

TABLE 6: KMO and Bartlett test.

\begin{tabular}{|c|c|c|}
\hline \multicolumn{2}{|c|}{ KMO sampling appropriateness number } & 0.931 \\
\hline & Approximate Chi-square & 3067.643 \\
\hline Bartlett sphericity test & Degree of freedom & 190 \\
\hline & Significance & 0.000 \\
\hline
\end{tabular}

TABLE 7: Factor matrix.

\begin{tabular}{|c|c|c|c|c|c|c|}
\hline \multicolumn{7}{|c|}{ Composition } \\
\hline & 1 & 2 & 3 & 4 & 5 & 6 \\
\hline A01 & 0.772 & & & & & \\
\hline $\mathrm{A} 02$ & 0.763 & & & & & \\
\hline A03 & 0.753 & & & & & \\
\hline A04 & 0.748 & & & & & \\
\hline A05 & 0.743 & & & & & \\
\hline A06 & 0.572 & & & & & \\
\hline A07 & & 0.730 & & & & \\
\hline A08 & & 0.704 & & & & \\
\hline A09 & & 0.594 & & & & \\
\hline A10 & & 0.562 & & & & \\
\hline A11 & & & 0.746 & & & \\
\hline A 12 & & & 0.717 & & & \\
\hline A13 & & & 0.552 & & & \\
\hline A14 & & & & 0.800 & & \\
\hline A 15 & & & & 0.754 & & \\
\hline A16 & & & & 0.533 & & \\
\hline A17 & & & & & 0.840 & \\
\hline A 18 & & & & & 0.776 & \\
\hline A19 & & & & & & 0.638 \\
\hline $\mathrm{A} 20$ & & & & & & 0.625 \\
\hline
\end{tabular}

Combined with the theoretical structure and factor analysis results, six factors were finally extracted.

\subsection{Experimental Hypothesis Validation Analysis}

4.3.1. Simulation Fitting Degree Analysis. This study mainly uses AMOS 26 to process structural equation model analysis. After modeling by AMOS, the measurement model of the structural equation model in this study included 6 latent variables, which were represented by ellipses. A total of 20 observed variables were represented by a square. There are
21 residuals, which were represented by circles. The final model is shown in Figure 3.

Table 8 shows the data of the overall fit degree of the model, which is analyzed from two aspects: absolute fit degree and value-added fit degree. It can be seen from the data in the table that the indexes of the two aspects of this model meet the evaluation criteria and the overall fitting degree is good. The structural equation model agrees well with the original data of the sample and meets the standard, indicating that the research model in Figure 3 can evaluate the research problem of influencing factors of Zhihu users' willingness to share knowledge. 


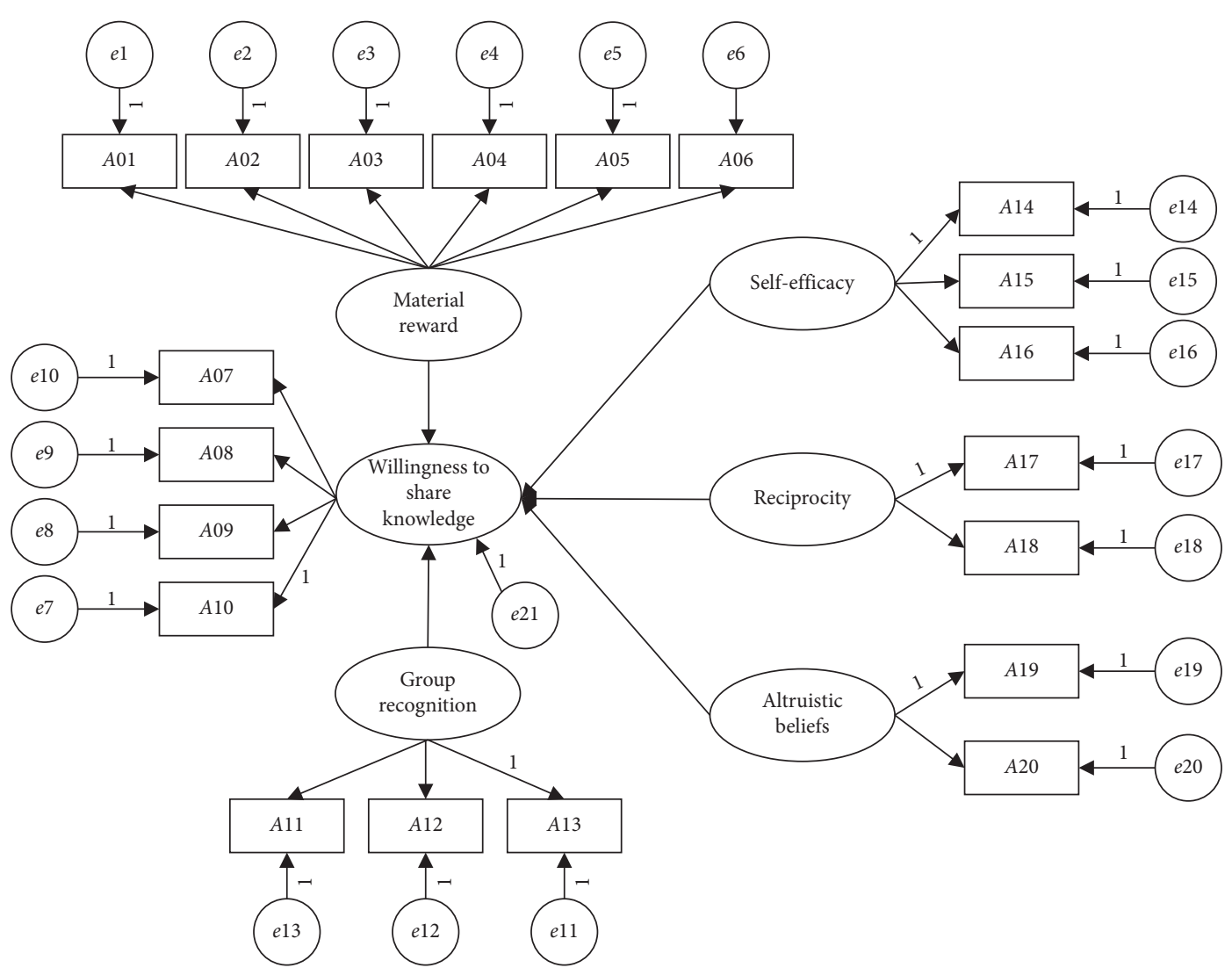

Figure 3: Structural equation model.

TABle 8: Model fit degree.

\begin{tabular}{|c|c|c|c|c|c|}
\hline & $\begin{array}{l}\text { Statistical test } \\
\text { volume }\end{array}$ & Name & $\begin{array}{l}\text { Adaptation } \\
\text { standard }\end{array}$ & $\begin{array}{c}\text { Test result } \\
\text { data }\end{array}$ & $\begin{array}{c}\text { Model adaptation } \\
\text { judgment }\end{array}$ \\
\hline \multirow{5}{*}{$\begin{array}{l}\text { Absolute adaptation } \\
\text { index }\end{array}$} & $\chi^{2} /$ d.f. & $\begin{array}{c}\text { Chi-square/degree of freedom } \\
\text { value }\end{array}$ & $<3$ & 1.733 & Yes \\
\hline & RMR & Residual root mean square & $<0.05$ & 0.025 & Yes \\
\hline & RMSEA & $\begin{array}{c}\text { Approximate residual root } \\
\text { mean square }\end{array}$ & $<0.08$ & 0.052 & Yes \\
\hline & GFI & Benign adaptation index & $>0.9$ & 0.913 & Yes \\
\hline & AGFI & $\begin{array}{l}\text { Adjusted benign adaptation } \\
\text { index }\end{array}$ & $>0.9$ & 0.877 & No \\
\hline \multirow{4}{*}{$\begin{array}{l}\text { Value-added adaptation } \\
\text { index }\end{array}$} & NFI & Rule adaptation index & $>0.9$ & 0.918 & Yes \\
\hline & RFI & Relative adaptation index & $>0.9$ & 0.896 & No \\
\hline & IFI & Value-added adaptation index & $>0.9$ & 0.964 & Yes \\
\hline & CFI & Comparative adaptation index & $>0.9$ & 0.963 & Yes \\
\hline
\end{tabular}

Wen et al. research [38] shows that as long as the model is a good fit according to several criteria (including Chisquare criterion), the model can be considered acceptable from some perspectives on the premise that other indexes should also be referred to and cannot be too far from the boundary value. Therefore, it can be considered that the fitting degree of this model is acceptable. At the same time, the maximum likelihood estimation method is used to estimate the parameters of the model, and the path analysis of the structural equation model is used to calculate the standardized path coefficients among the potential variables. In addition, the standardized path regression coefficients among model variables are studied in this paper to more intuitively explain the results of hypothesis verification of this research (Figure 4).

4.3.2. Hypothesis Test Result. It can be seen from Tables 8 and 9 that the hypotheses proposed in this paper are partially valid. The experimental results of the knowledge-sharing willingness research model constructed in this paper show that the path coefficients of self-efficacy and material rewards are positive and the significant $p$ value is high, so they have a positive impact on the knowledge-sharing willingness, while 


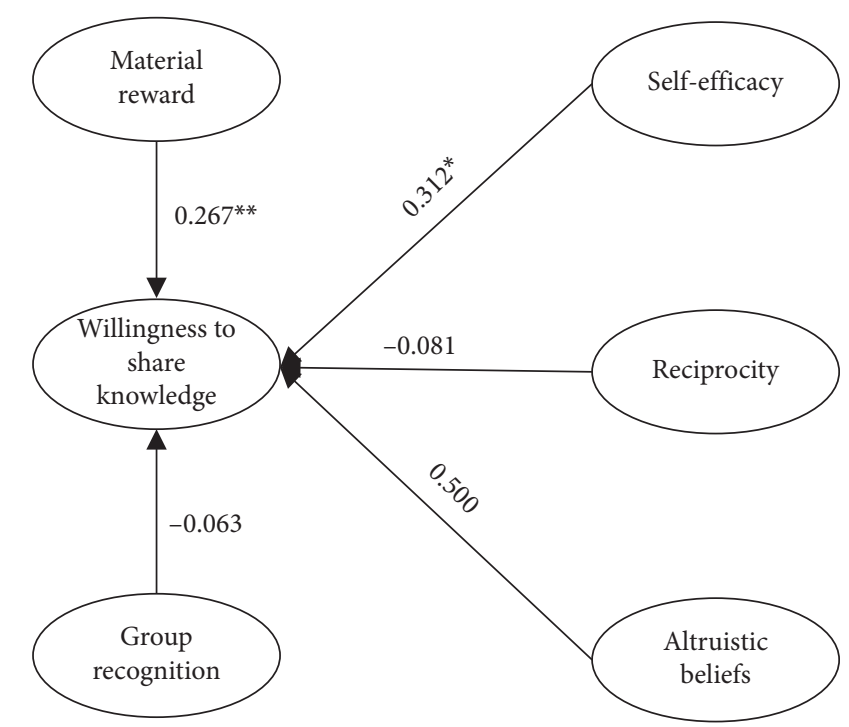

Figure 4: Structural equation model analysis results. Note: ${ }^{*} p<0.05,{ }^{* *} p<0.01$, and ${ }^{* * *} p<0.001$.

TABle 9: Assumption verification result.

\begin{tabular}{lcccc}
\hline Hypothesis & Verification path & Path coefficient & $p$ value & Validation results \\
\hline H1 & Reciprocity $\longrightarrow$ knowledge-sharing willingness & -0.081 & 0.374 & Invalid \\
H2 & Group recognition $\longrightarrow$ knowledge-sharing willingness & -0.063 & 0.810 & Invalid \\
H3 & Self-efficacy $\longrightarrow$ knowledge-sharing willingness & 0.312 & 0.050 & Valid \\
H4 & Altruistic belief $\longrightarrow$ knowledge-sharing willingness & 0.500 & 0.182 & Invalid \\
H5 & Material reward $\longrightarrow$ knowledge-sharing willingness & 0.267 & 0.005 & Valid \\
\hline
\end{tabular}

reciprocity and group recognition have no positive effect on knowledge-sharing willingness. According to the data, although altruistic beliefs have an impact on the willingness to share knowledge, the significance and $p$ value are not high, which does not indicate that it has a positive effect on the willingness to share knowledge.

\section{Conclusions}

Based on the literature research and the basic situation of Zhihu's use, this study proposes the influencing factor model of Zhihu users' willingness to share knowledge based on the theory of planned behavior. The knowledge-sharing willingness of Zhihu users was studied through multiple factors, 271 pieces valid data from Zhihu users were collected by using questionnaires, and the research hypotheses were verified by using the structural equation model to verify the influence of reciprocity, social capital, group recognition, self-efficacy, and altruistic beliefs on the knowledge-sharing willingness. The research results show that self-efficacy and material rewards have a positive effect on the knowledgesharing willingness, altruistic beliefs are not significant, and reciprocity and group recognition have negative effects.

Since the respondents are all representative, young people between the ages of 20 and 30 occupy most of the power of interpretation in this study. Because the respondents are mainly randomly selected college students, the accuracy of the proportion of young people in the age characteristics of the questionnaire data can be determined, which covers the main user groups of Zhihu users. Compared with the continuous development of Zhihu users and the continuous penetration of new and old users, the sample data in this study is more representative of the knowledgesharing willingness of some young users. Data analysis results show that most young people at present are more inclined to the positive impact of self-efficacy and material rewards, which shows that many young people in society now hope to get some opportunities to prove their social value and are good at sharing their own experiences. However, because, in this era of information sharing, a lot of knowledge has been transported and cannot reflect the value of the sharer, young people now also hope more to get some external rewards in return for their efforts. This is because high-quality knowledge sharing requires the sharers to accumulate in the early stage and even invest a lot of time and energy, and material rewards can compensate and motivate the sharers. In this regard, today's knowledge community platforms must not only give sharers the opportunity to show their self-worth, but also learn to use appropriate rewards and incentives for users to share knowledge in order to maintain the operation of the community platform.

The three influencing factors of altruistic beliefs, reciprocity, and group recognition do not have a positive effect on the knowledge-sharing willingness, but relevant literature has confirmed that these three influencing factors have a positive effect on the knowledge-sharing willingness. Research shows that the data in this questionnaire is not rigorous enough or that Zhihu's new and old users are 
constantly changing. Users of different age groups have different perceptions of Zhihu. The research results only represent the behavioral willingness of the group we studied. Therefore, the impact of the three influencing factors of altruistic beliefs, reciprocity, and group recognition on the knowledge-sharing willingness, as well as the knowledgesharing behavior of different groups, remains to be investigated.

\section{Data Availability}

The data presented in this study are available on request from the corresponding author.

\section{Conflicts of Interest}

All authors declare that there are no conflicts of interest regarding the publication of this paper.

\section{Acknowledgments}

The authors acknowledge the financial support from the National Natural Science Foundation of China (no. 11905042), 333 Funded Project of "333 Talent Project" in Hebei Province (no. A202001015), and Key Project of Humanities and Social Sciences Research for the Colleges and Universities of Hebei Province (no. SD2021017).

\section{References}

[1] F. M. Harper, D. Raban, and S. Rafaeli, "Predictors of answer quality in online Q\&A sites," in Proceedings of the SIGCHI Conference on Human Factors in Computing Systems, pp. 865-874, ACM, Florence, Italy, April 2008.

[2] N. Choi and K. Yi, "Raising the general public's awareness and adoption of open source software through social Q\&A interactions," Online Information Review, vol. 39, no. 1, pp. 119-139, 2015.

[3] R. Cronk, "Knowledge sharing in the online environment: emotional intelligence, social capital, and intellectual capital relationships," in Proceedings of the European Conference On Knowledge Management, pp. 232-239, Barcelona, Spain, September 2017.

[4] S. Alexander and B. Nick, "Negotiate, reciprocate, or cooperate? The impact of exchange modes on inter-employee knowledge sharing," Journal of Knowledge Management, vol. 20 , no. 4, pp. 687-712, 2016.

[5] H. S. Meng, L. Teresa, and M. C. Chun, "Knowledge sharing in virtual communities: the relationship between trust, self-efficacy and outcome expectations," International Journal of Human-Computer Studies, vol. 65, no. 2, pp. 153-169, 2007.

[6] F. Hassandoust, M. F. Kazerouni, and V. Perumal, "Sociobehavioral factors in virtual knowledge sharing," International Journal of Knowledge-Based Organizations, vol. 2, no. 2, pp. 40-53, 2012.

[7] Y. L. Chen, N. S. Chen, and Kinshuk, "Examining the factors influencing participants' knowledge sharing behavior in virtual learning communities," Journal of Educational Technology \& Society, vol. 12, no. 1, pp. 134-148, 2009.

[8] Z. Tao, "Explaining virtual community user knowledge sharing based on social cognitive theory," Wireless
Communications Networking \& Mobile Computing-WiCOM, vol. 31, pp. 9538-9541, 2008.

[9] Y. F. Liu and F. F. Jia, "Research on user knowledge-sharing behavior based on SNS," Information Science, vol. 35, no. 1, pp. 41-46, 2017.

[10] R. Liu, P. Tian, and W. J. Wang, "An empirical study on influencing factors of knowledge-sharing in virtual communities in Chinese cultural context," Information Science, vol. 30, no. 6, pp. 866-872, 2012.

[11] Z. R. Fu, Research On Knowledge Sharing Attitudes Of Information Personnel in Organizations-Multilevel Analysis Model Method, Central University, Taiwan, Taoyuan, 2005.

[12] I. Ajzen, "Perceived behavioral control, self-efficacy, locus of control, and the theory of planned behavior," Journal of Applied Social Psychology, vol. 32, no. 4, pp. 665-683, 2002.

[13] M. R. Keats, S. N. Culos-Reed, K. S. Courneya, and M. McBride, "Understanding physical activity in adolescent cancer survivors: an application of the theory of planned behavior," Psycho-Oncology, vol. 16, no. 5, pp. 448-457, 2007.

[14] T. Zahra and M. Mohammad, "Knowledge sharing behaviour and its predictors," Industrial Management \& Data Systems, vol. 110, no. 4, pp. 611-631, 2010.

[15] C.-C. Chen, "Factors affecting high school teachers' knowledge-sharing behaviors," Social Behavior and Personality: An International Journal, vol. 39, no. 7, pp. 993-1008, 2011.

[16] O. W. Bello and R. A. Oyekunle, "Attitude, perceptions, motivation towards knowledge sharing: views from universities in kwara state," African Journal of Library, vol. 24, no. 2, pp. 123-134, 2014.

[17] H. Jin, Z. Yang, and F. Feng, "Research on material incentives, knowledge ownership and organizational knowledge sharing," Science Research, vol. 29, no. 7, pp. 1036-1045, 2011.

[18] W. J. Zhao, "Research on Sustainable Behavior of Knowledge Sharing in Virtual Community," Central China Normal University, Hubei, China, 2012.

[19] O. Pilerot, "Information sharing in the field of design research," Information Research: An International Electronic Journal, vol. 20, no. 1, pp. 26-45, 2015.

[20] C. Tong, W. I. W. Tak, and A. Wong, "The impact of knowledge sharing on the relationship between organizational culture and job satisfaction: the perception of information communication and technology (ICT) practitioners in Hong Kong," International Journal of Human Resource Studies, vol. 5, no. 1, pp. 19-47, 2015.

[21] Q. Hao, C. Jin, and K. Wei, "The influence mechanism of knowledge-sharing behavior of virtual team members: the perspective of personal and environment interaction," Technological Progress and Countermeasures, vol. 36, no. 7, pp. 138-144, 2019.

[22] C. M. L. Chan, M. Bhandar, H.-C. Chan, and L.-B. Oh, "Recognition and participation in a virtual community," in Proceedings of the 37th Annual Hawaii International Conference On System Sciences, p. 10, Big Island, HI, USA, February 2004.

[23] J. Koh and Y.-G. Kim, "Sense of virtual community: a conceptual framework and empirical validation," International Journal of Electronic Commerce, vol. 8, no. 2, pp. 75-94, 2003.

[24] Z. B. Yang, "Research on influencing factors and interaction of knowledge-sharing in socialized question-and-answer website," Management Case Studies and Reviews, vol. 9, no. 3, pp. 212-223, 2016.

[25] H. J. Yang, "An empirical study on the influencing factors of users' willingness to contribute to social Q\&A," Library Science Research, vol. 14, pp. 29-38, 2014. 
[26] M. A. Fishbein and I. Ajzen, Belief, Attitude, Intention and Behavior: An Introduction to Theory and Research, Reading, MA: Addison-Wesley, Boston, MA, USA, 1975.

[27] Z. Zhang, An Empirical Study on the Influencing Factors of Individual Knowledge-Sharing in the Mobile Internet Environment, University of Science and Technology of China, Hefei, China, 2015.

[28] X. L. Wang, Q. Y. Zhang, and Q. Liu, "Path analysis of the influence of knowledge-sharing and knowledge innovation on organizational performance," Journal of Inner Mongolia University of Technology (Natural Science Edition), vol. 33, no. 4, pp. 315-319, 2014.

[29] P. M. Blau and G. W. Li, Exchange and Power in Social Life, Commercial Press, Beijing, China, 2008.

[30] T. Connolly and B. K. Thorn, Discretionary Databases: Theory, Date and Implications, Sage Publications, New York, NY, USA, 1990.

[31] G. W. Bock and Y.-G. Kim, "Breaking the myths of rewards," Information Resources Management Journal, vol. 15, no. 2, pp. 14-21, 2002.

[32] H.-F. Lin, "Effects of extrinsic and intrinsic motivation on employee knowledge sharing intentions," Journal of Information Science, vol. 33, no. 2, pp. 135-149, 2007.

[33] P. Runhaar and K. Sanders, "Promoting teachers' knowledge sharing: the fostering roles of occupational self-efficacy and human resources management," Educational Management Administration \& Leadership, vol. 27, no. 5, pp. 1-20, 2014.

[34] M.-H. Hsu, T. L. Ju, C.-H. Yen, and C.-M. Chang, "Knowledge sharing behavior in virtual communities: the relationship between trust, self-efficacy, and outcome expectations," International Journal of Human-Computer Studies, vol. 65, no. 2, pp. 153-169, 2007.

[35] C. M. Gan, W. J. Wang, and P. Tian, "Research on the psychological incentives of knowledge exchange and sharing in academic blogs," Journal of Library Science in China, vol. 38, no. 3, pp. 91-99, 2012.

[36] Y. F. Shen, B. Liao, and Y. Xu, "Research on the influence of reputation system on knowledge sharing activities in social Q\&A communities," Journal of Information, vol. 37, no. 11, pp. 1154-1163, 2018.

[37] B. A. Cerny and H. F. Kaiser, "A study of a measure of sampling adequacy for factor-analytic correlation matrices," Multivariate Behavioral Research, vol. 12, no. 1, pp. 43-47, 1977.

[38] Z. L. Wen, J. T. Hou, and H. Marsh, "Structural equation model testing: fitting index and chi-square criterion," Psychological News, vol. 2, pp. 186-194, 2004. 